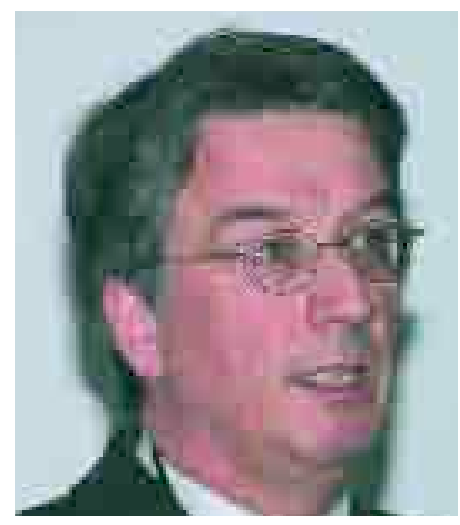

\section{EBM: Durch Fakten oder Annahmen? Gedanken zum Lancet-Artikel über Homöopathie}

„Evidenz" liefert ein Urteil nur dann und nur insofern, als es sich auf tatsächlich vorhandene Fakten bezieht. Urteile und Schlussfolgerungen, die auf Annahmen beruhen, bieten keine Evidenz, auch wenn sie klug berechnet sind. Das gilt auch für die Evidenz basierte Medizin, EBM, wie man sehr gut am Beispiel der Lancet-Studie über Homöopathie von SHANG et al. aus dem Berner Institut für Sozial- und Präventivmedizin von Prof. Egger sehen kann [1]. Prof. Egger gilt als prominenter Vertreter von EBM. Seine Arbeitsgruppe kommt auf Grund einer MetaRegression bekanntlich zum Schluss, dass Homöopathie nicht wirkungsvoller sei als Placebo. Bezieht sich dieses Urteil tatsächlich auf nachgewiesene Fakten? Nein. Die wesentlichen Schlussfolgerung sind Hypostasen, die auf theoretischen Annahmen und darauf aufbauenden probabilistischen Berechnungen beruhen.

1. Es wird angenommen, dass die Effekte der untersuchten Homöopathie-Studien homogen seien, denn nur unter dieser Voraussetzung ist das Herzstück der Studie, die gewählte Funnel Plot Methode sinnvoll. In Wirklichkeit umfassen die Studien jedoch unterschiedlichste Studienmodelle und homöopathische Vorgehensweisen, so dass homogene Effektarten nicht zu erwarten sind. Bei inhomogenen Effektarten kann der Funnel Plot ähnlich schief werden wie in der vorliegenden Arbeit, auch ohne dass ein Publication Bias vorliegt [2]. Das ist bereits im Schlussbericht PEK im Hinblick auf Eggers Arbeit kritisert worden [3].

2. Es wird von der Egger-Gruppe aus rein theoretischen Gründen angenommen, dass der schiefe Funnel Plot auf einem Publication Bias beruhe, ohne faktischen Nachweis. Dem Publication Bias sind jedoch schon LINDE et al. in ihrer Meta-Analyse von 1997 genauer nachgegangen, indem sie systematisch nach nicht-publizierten Studien suchten und ihre Berechnungen auch für einen hypothetischen Publication Bias adjustierten. Dadurch verminderte sich der gepoolte Effekt der Studien, er blieb aber immer noch signifikant positiv zu Gunsten der Homöopathie. Es wären je nach Berechnung 923 oder 4511 (!) „fehlende“ Studien mit einem Null-Effekt nötig gewesen, um den Gesamteffekt unter die Signifikanzgrenze zu senken [ 2]. Es gibt aber weltweit nicht genügend Arbeitsgruppen, um so viele Studien zustande zu bringen und sie erst noch zu verstecken. Im übrigen zeigen auch die meisten von LINDE et al. gefundenen nicht-publizierten Studien einen positiven Effekt.

3. Es wird rein theoretisch vorausgesetzt, dass die grossen Studien qualitativ besser seien als die kleineren. Die faktische Evidenz dafür wurde nicht geprüft. Bei der Klassischen Homöopathie ist eher das Gegenteil der Fall, nämlich dass ihre individualisierende Vorgehensweise in einer grossen Studie nicht gewährleistet ist oder dass nicht genügend kompetente Therapeuten zu Verfügung stehen. Falsch negative Ergebnisse sind dann die Folge. Für konventionelle Therapien gilt wegen ihrer weitgehenden Standardisierung und der viel weiter gehenden Unabhängigkeit der Medikamentengabe von der spezifischen Kompetenz der Ärzte nicht dasselbe. Das von Shang et al. benutzte statistische Argument der grossen Zahl ist deshalb nicht sehr aussagekräftig. Zudem blieben bei der Qualitätsfrage alle Gesichtspunkte der externen Validität ausgeklammert, was jedoch bei der Homöopathie und anderen komplementärmedizinischen Methoden nicht zulässig ist [4].

4. Das Urteil, dass die Homöopathie nicht wirkungsvoller sei als Placebo und dass dies für konventionelle Medizin nicht zutreffe, wird letztlich auf Grund einer Selektion von 8 (!) der grösseren und qualitativ besseren Homöopathiestudien und von 6 (!) entsprechenden konventionellen Studien sowie aufgrund einer rein statistischen Extrapolation auf virtuelle (!) grosse Studien gefällt. Die 8 bzw. 6 Studien sind nicht referenziert, es werden keine Selektionskriterien für ihre Auswahl angegeben. Es wird vom Faktum abgesehen, dass in einem Grossteil der 110 Studien die Homöopathie dem Placebo überlegen war und dass die meisten früheren Übersichtsarbeiten und Metaanalysen ein positives Gesamtergebnis für die Homöopathie ergeben hatten [5-9]. D.h. die gesamte vorliegende Evidenz wird disqualifiziert. Aber die Autoren offenbaren hier klar, welchem Bias sie selbst unterliegen. So geben sie für 8 Homöopa- 
thiestudien bei akuten oberen Luftwegsinfekten einen hohen gepoolten Effekt zu, der auch der Funnel Plot Analyse und ihrem Qualitätskriterium standhielt, was eigentlich als „robust evidence" für die Wirksamkeit der Therapie gelten müsste. "However", schreiben sie, "the biases that are present in these publications, as shown in our study, might promote the conclusion that the results cannot be trusted" (Herv o rhebungen P.H.). Die Autoren belegen damit, dass sie ihre theoretische Annahme eines nota bene riesigen Publication Bias und das Resultat ihrer rein probabilistischen Berechnungen als ein von ihnen aufgezeigtes Faktum hinstellen und so den tatsächlich vorliegenden Fakten misstrauen. Warum? Weil die Homöopathie offenbar der gewohnten Theorie über das Wesen der Substanz widerspricht, wie auch VANDENBROUCKE in seinem Kommentar offen zugibt: „We question the results of a randomised trial of homoeopathy because we know that pharmacological action of infinite dilutions is highly implausible. This reasoning is also the explicit starting point of Shang and colleagues and their analysis only gain meaning because of that background"[10].

Hier also der motivierende Hintergrund-Bias: eine pharmakologische Theorie, die bekanntlich auf Modellvorstellungen (!) der Materie beruht. Die Fakten werden sich jedoch um solche Theorien, Annahmen und Berechnungen wenig kümmern. Daher zeigt schon die nächste randomisierte Placebo-kontrollierte cross-over Studie unserer Berner Kollegen, dass Homöopathie trotzdem wirkt, diesmal bei Kindern mit Aufmerksamkeitsstörungen und Hyperaktivität [11].

\section{Literatur}

1. Shang A, Huwiler-Müntener K, Nartey L, et al.: Are the clinical effects of homoeopathy placebo effects? Comparative study of placebo-controlled trials of homoeopathy and allopathy. Lancet 2005; 366: 726-32

2. Linde K, Clausius N, Ramirez G, et al. : Are the clinical effects of homoeopathy placebo effects? A meta-analysis of placebo-controlled trials. Lancet 1997; 350: 834-43

3. Melchart D, et al. Schlussbericht Programm Evaluation Komplementärmedizin PEK. 1 Vesion Februar 2005 und Schlussversion 24.4.2005. Bundesamt für Gesundheit, Bern

4. Heusser P: Kriterien zur Beurteilung des Nutzens von komplementärmedizinischen Methoden. Forsch Komplementärmed Klass Naturheilkd 2001; 8: 14-23

5. Kleijnen J, Knipshild P, ter Riet G: Clinical trials of homoeopathy. BMJ 1991; 302:316-23

6. Boissel J: Critical literature review on the effectiveness of homoeopathy: overview over data from homoeopathy medicine trials. In: Commission of the European Communities (ed.) Homoeopathic Medicine Research Group. Commission of the European Communities 1996, p.196-210

7. Cucherat $\mathrm{M}$ et al: Evidence of clinical efficacy of homoeopathy. A meta-analysis of clinical trials. Homoeopathic Medicines Research Advisory Group HMRAG. Eur J Clin Pharmacol 2000; 56: 27-33

8. Wein C : Qualitätsaspekte klinischer Studien zur Homöopathie. KVC, Essen 2002

9. Mathie RT: The research evidence base for homoeopathy: a fresh assessment of the literature. Homoeopathy: The Journal of the Faculty of Homoeopathy 2003; 92 (2): 84-89

10. Vandenbroucke JP: Homoeopathy and "the growth of truth". Lancet 2005; 366: 691-92

11. Frei $H$, Everts $R$, von Ammon $K$, et al: Homoeopathic treatment of children with attention deficit hyperactivity disorder: a randomised, double blind, placebo controlled crossover trial. European Journal of Pediatrics 2005. On-line publication Springer Verlag, 10.1007/s00431-005-1735-7

Univ. Doz. Dr. med. Peter Heusser, KIKOM, Universität Bern 